\title{
Practical procedure for discriminating monofloral honey with a broad pollen profile variability using an electronic tongue
}

\author{
Mara E.B.C. Sousa ${ }^{a}$, Luís G. Dias ${ }^{\mathrm{a}, *}$, Ana C.A. Veloso ${ }^{\mathrm{b}, \mathrm{c}}$, Letícia Estevinho ${ }^{\mathrm{a}}$, \\ António M. Peres ${ }^{\mathrm{a}, \mathrm{d}}$, Adélio A.S.C. Machado ${ }^{\mathrm{e}}$ \\ ${ }^{a}$ CIMO - Mountain Research Centre, Escola Superior Agrária, Instituto Politécnico de Bragança, Campus Santa Apolónia, \\ Apartado 1172, 5301-855 Bragança, Portugal \\ b Instituto Politécnico de Coimbra, ISEC, DEQB, Rua Pedro Nunes, Quinta da Nora, 3030-199 Coimbra, Portugal \\ ${ }^{\mathrm{C}}$ CEB - Centre of Biological Engineering, University of Minho, Campus de Gualtar, 4710-057 Braga, Portugal \\ ${ }^{\mathrm{d}}$ LSRE - Laboratory of Separation and Reaction Engineering - Associate Laboratory LSRE/LCM, Escola Superior Agrária, Instituto Politécnico de Bragança, \\ Campus Santa Apolónia, Apartado 1172, 5301-855 Bragança, Portugal \\ ${ }^{\mathrm{e}}$ LAQUIPAI - Laboratório de Química Inorgânica Pura e de Aplicação Interdisciplinar, Departamento de Química, Faculdade de Ciências da Universidade do \\ Porto, R. Campo Alegre, 687, 4169-007 Porto, Portugal
}

\section{A R T I C L E I N F O}

\section{Article history:}

Received 10 January 2014

Received in revised form

28 April 2014

Accepted 4 May 2014

Available online 10 May 2014

Keywords:

Electronic tongue

Monofloral honey

Honey colour

Linear discriminant analysis

Simulated annealing algorithm

\begin{abstract}
A B S T R A C T
Colour and floral origin are key parameters that may influence the honey market. Monofloral light honey are more demanded by consumers, mainly due to their flavour, being more valuable for producers due to their higher price when compared to darker honey. The latter usually have a high anti-oxidant content that increases their healthy potential. This work showed that it is possible to correctly classify monofloral honey with a high variability in floral origin with a potentiometric electronic tongue after making a preliminary selection of honey according their colours: white, amber and dark honey. The results showed that the device had a very satisfactory sensitivity towards floral origin (Castanea sp., Echium sp., Erica sp., Lavandula sp., Prunus sp. and Rubus sp.), allowing a leave-one-out cross validation correct classification of $100 \%$. Therefore, the E-tongue shows potential to be used at analytical laboratory level for honey samples classification according to market and quality parameters, as a practical tool for ensuring monofloral honey authenticity.
\end{abstract}

(c) 2014 Elsevier B.V. All rights reserved.

\section{Introduction}

The term honey can be related to different food products taking into account the raw material and their floral origin, namely honeydew, monofloral or polyfloral honey. Also, honey is usually classified considering its colour. Colours of honey cover a continuous range from very pale yellow through ambers to nearly black, which may be attributed mainly to the plant source of the honey $[1,2]$. From a consumer's point of view, the colour is a key factor when purchasing, since honey colour is reported to be a factor in its grading and marketing, lighter colour honey being associated with more smooth flavour and in general more expensive [3-5]. On the other hand, monofloral honey is also much more valuable from a commercial point of view, due to their higher market prices, when compared namely to polyfloral honey [6-8]. Therefore, colour and floral classifications of honey samples are of major economic importance for both producers and consumers. Colour-

\footnotetext{
* Corresponding author. Tel.: +351 273303323; fax: + 351273325405.

E-mail address: Idias@ipb.pt (L.G. Dias).
}

Pfund classification is usually based on UV/vis spectrophotometric analysis, results of which are later related to a colour scale, considered a simple methodology. On the other hand, floral honey classification, is a time-consuming task that requires high-skilled technicians, since it is mainly based on melissopalynological analysis, which in some cases must be complemented by sensory analysis, as for some types of honey the interpretation of melissopalynological analysis can be complicated and sometimes imprecise and ambiguous [6]. Even so, Corbella and Cozzolino [9] reported the combination of multivariate techniques and pollen count analysis to classify honey samples from Uruguay according to botanical sources, although not in the industrial context. Therefore, novel, fast and low-cost practical tools that could be easily applied to the analysis of the majority of honey samples are needed and, in fact, even the EU commission encourages the development of new methods for honey authentication, as pointed out by some researchers [10].

In the last years several studies have dealt the honey authentication problematic by applying multivariate statistical techniques to different physicochemical data (such as colour, diastase activity, water content, ash, free amino acids, lactone and total acidity, $\mathrm{pH}$, 
electrical conductivity, viscosity, total antioxidant activity, flavonoids and phenolic acids) [7,11-14] or to honey volatile fraction measurements [15-17], among other techniques reported in literature for geographical and botanical classification of honey $[8,18,19]$. In general, all these approaches showed good discrimination capabilities, precision, accuracy and reliability, but they are in general destructive, time-consuming and expensive, being unsuitable for in situ monitoring [10]. To overcome these drawbacks other more simple and userfriendly methodologies have been proposed, namely the use of potentiometric $[6,10,21,22]$, voltammetric $[23,24]$ or impedance [25] electronic tongues (E-tongues). The results reported in these studies clearly show that all these electrochemical devices can be used as effective and practical tools to discriminate honey according to their botanical origin, allowing distinguishing among different monofloral and/or polyfloral samples and in some cases among different geographical origins.

With reference to the potentiometric devices, the first approach was published by Dias et al. [20], where an E-tongue, containing all-solid-state potentiometric sensors with polymeric membranes, together with multivariate chemometric tools (principal component analysis, PCA; and linear discriminant analysis, LDA), being developed and applied in the classification of 52 commercial Portuguese honey samples according to their botanical origin, namely as Lavandula, Erica or Echium monofloral honey (around $70 \%$ of correct classifications). Wei et al. [21] used a commercial potentiometric E-tongue with 7 sensors to classify 192 Chinese honey samples of different floral origin (Acacia, Astragali, Buckwheat, Coptis, Data, Motherwort, Radix Changll and Vitex) from a Chinese geographical area and 120 samples from five other Chinese geographical origins, harvested in 2008, using threepattern recognitions techniques (PCA; Cluster Analysis, CA; and Artificial Neural Networks, ANN), with correct classification rates greater than $90 \%$. Zakaria et al. [22] showed that an electronic nose used together with a chalcogenide-based potentiometric E-tongue containing ion-selective sensors, was the best solution for classifying honey of different floral origin. In this study, 80 honey samples from 10 monofloral honey from Malaysia and New Zealand and 4 brands of polyfloral honey from Malaysia were used. The sensor fusion methodology allowed a $100 \%$ correct classification for crossvalidation procedure using a LDA approach and over than $90 \%$ using ANN for an external validation group. Major et al. [10] used a commercial potentiometric E-tongue, comprised of 7 sensors, for botanical classification and physicochemical characterisation of honey samples (acacia, chestnut and honeydew honey) from Croatia. Using ANN models, all training and testing samples were correctly classified according to their botanical origin. Escriche et al. [6] proposed a potentiometric E-tongue, made of metals and metallic compounds that, together with ANN, was able to correctly classify the botanical origin of 32 Spanish honey samples harvested in 2008 and 2009 with a success greater than 90\% for three honey of floral origin (citrus, rosemary monofloral and polyfloral) and one honeydew (forest origin). Furthermore, a good correlation was observed between the E-tongue and colour-Pfund, luminosity and diastase activity. More recently, this same research team [26] used the same potentiometric device to satisfactorily classify honey samples (with a correct recognition greater than 75\%) according to their botanical origin (citrus, rosemary and polyfloral) and physical treatment, usually applied to commercial honey (raw, liquation and pasteurisation), using algorithms based on Fuzzy ARTMAP simplified artificial neural networks.

Therefore, considering: (i) the encouraging results achieved so far by different groups regarding honey botanical origin classification using potentiomettric E-tongues coupled with chemometric tools, and (ii) the commercial interest in classifying honey according to botanical origin, in this work a new classification strategy was evaluated envisaging the correct classification of samples that greatly vary in colour and pollen profile, which will be the normal practical case, using an E-tongue.

To the best of author's knowledge, an E-tongue has never been applied before to classify honey samples with the wide variability in colour and floral origin that was evidenced in the analysed samples. For that purpose a new all-solid-state potentiometric E-tongue device was constructed, using a print-screen technique, comprising cross-sensitivity sensors. The analysis carried out included a broader database than that previously used [20], namely, 65 monofloral Portuguese honey samples harvested in 3 consecutive years (from 2009 up to 2011) from all regions of beekeeping production of the mainland of Portugal, resulting in 6 different floral origins, according to pollinic analysis. E-tongue data were analysed using a two-step sequential procedure. First, a LDA model was established based on the potentiometric signal sensor profiles selected using a meta-heuristic variable selection algorithm, to show that it is possible to discriminate honey samples according to their colours (white, amber and dark honey, which were classified according to a colour-Pfund method). Finally, the main purpose, for each colour group selected, was to classify honey samples considering their floral origin by using a LDA based on the E-tongue data after variable selection.

\section{Materials and methods}

\subsection{Honey samples}

Sixty five monofloral Portuguese honey samples, harvested between 2009 and 2011, were kindly disposed by the Federação Nacional dos Apicultores de Portugal (FNAP), being a representative sampling of the most productive Portuguese honey regions, both in the continent (e.g., Trás-os-Montes e Alto Douro; Entre Douro e Minho; Beira Interior; Beira Litoral; Estremadura e Ribatejo; Alentejo; and Algarve regions) and Azores (Pico and São Miguel islands). Samples were stored at room temperature until analysis. Each sample was split for colour evaluation, floral origin classification based on melissopalynological assays and potentiometric signal profile analysis with an E-tongue device.

\subsection{Honey colour classification}

The colour of each honey sample was evaluated using a quantitative millimetre Pfund ( $\mathrm{mm}$ Pfund) scale [27]. The $\mathrm{mm}$ Pfund values were calculated from the absorbance of diluted honey samples ( $5.0 \mathrm{~g}$ of honey in $10.0 \mathrm{~mL}$ of deionised water) recorded at $635 \mathrm{~nm}$ measured with an UV/vis spectrophotometer (Jenway, Genova model), according to [27]

$\mathrm{mm}$ Pfund $=-3870+37,139 \times$ Absorbance

The quantitative mm Pfund scale was transformed into a qualitative colour classification according to the scale defined by the United States Department of Agriculture [28], which considers 7 levels of colour for honey: water white ( $\leq 8 \mathrm{~mm}$ Pfund), extra white $(8<\mathrm{mm}$ Pfund $\leq 17)$, white $(17<\mathrm{mm}$ Pfund $\leq 34)$, extra light amber $(34<\mathrm{mm}$ Pfund $\leq 50)$, light amber $(50<\mathrm{mm}$ Pfund $\leq 85)$, amber $(85<\mathrm{mm}$ Pfund $\leq 114)$ and dark amber $(>114 \mathrm{~mm}$ Pfund). Considering a broader colour classification scale, honey samples were split into only 3 main colour groups: white $(\mathrm{mm}$ Pfund $\leq 34)$, amber $(34<\mathrm{mm}$ Pfund $\leq 114)$ and dark ( $>114 \mathrm{~mm}$ Pfund) colours. This colour grouping was chosen considering the dimension of database used and the main colour groups that are naturally identified in honey samples. 


\subsection{Melissopalynology analysis of honey}

The honey pollen quantitative spectrum analysis was performed according to the method reported by Louveaux et al. [29]. For each analysis, $10 \mathrm{~g}$ of honey were diluted with $30 \mathrm{~mL}$ of distilled water and the sediment was concentrated by centrifugation at $1500 \mathrm{rpm}$ during $30 \mathrm{~min}$. To the recovered sediment an addition of $10.0 \mathrm{~mL}$ of anhydride acetic (Panreac) and sulphuric acid (M\&B; 9:1, v/v) was made. After incubation in a water bath ( $100{ }^{\circ} \mathrm{C}$ during $3 \mathrm{~min}$ ) with agitation, a new centrifugation was carried out and the solution was decanted. Then, $12.0 \mathrm{~mL}$ of acetic acid (Merck) were added to the sediment and, after agitation, a new centrifugation and decantation were made. The sediment was washed and re-suspended in $12.0 \mathrm{~mL}$ of distilled water, and centrifuged and decanted again. The final wash was made with $12.0 \mathrm{~mL}$ of $\mathrm{KOH} 7 \%$ (Merck) solution, and a repetition of the agitation, centrifugation and decantation steps was accomplished. Finally, the pollen grains were stained using a fuchsin solution (Merck) mixed with glycerine (Absolve).

Pollen identification and count were carried out using an optic microscope (Leitz Messtechnik GmbH, Wetzlar, Germany) with $400 \times$ and $1000 \times$ objectives (the last one was used when greater detail was required for pollen identification). For each honey sample, a minimum of 1000 grains of pollen was counted, and in case of doubt the analysis was repeated. Reference standards obtained from Portugal honey flora (available at Escola Superior Agrária - Instituto Politécnico de Bragança, Portugal) were used for grain pollen identification and the samples were classified based on their floral origin according to their found pollen morphology.

\subsection{E-tongue analysis}

\subsubsection{Multisensor system}

The multisensor system was printed in both sides of a PVC board using a print-screen technique, by applying an epoxy conductive silver paste (EPO-TK E4110, Epoxy Technology, Inc.) with low temperature curing, prepared by mixture of two reagents (paste and hardener). In each side of the system, 10 chemical sensors can be applied. The system was cured at $40{ }^{\circ} \mathrm{C}$ allowing obtaining a dried paste in $8 \mathrm{~h}$. After cutting and cleaning the impressed circuit, the system was waterproofed using an acrylic resin (PLASTIK 70, da Kontakt Chemie). During the waterproofing, the contact spots where polymeric membranes would be applied as well as the connection section where the RS-232 pin 25 male plug will be connected, were protected. Fig. 1 shows an example of a multi-sensor system showing black covers protecting the spots where polymeric membranes will be applied. Before use, each system was tested with a multimeter (Digital Multimeter UniVolt DT-64) to verify the conduction of the electrical signal.

\subsubsection{Chemical sensors}

The two multi-sensor arrays constructed used different cross-sensitivity membranes as chemical sensors, with different pre-established mass combinations of 4 lipidic additives (octadecylamine, oleyl alcohol, methyltrioctylammonium chloride and oleic acid from Fluka; between 2.8 and 3.2\%), 5 plasticizers (bis (1-butylpentyl) adipate, dibutyl sebacate, 2-nitrophenyl-octylether, tris(2-ethylhexyl)phosphate and dioctyl phenylphosphonate, from Fluka; between $64.7 \%$ and 65.2\%) and PVC high molecular weight polymer (poly(vinyl chloride) polymer, between $31.9 \%$ and $32.3 \%$ ), as shown in Tables 1 and 2, identified with a code with a letter $\mathrm{S}$ (for sensor) followed by the number of the array (1 or 2 ) followed by the number of the membrane (1-20, corresponding to different combinations of plasticizer and additive used). The membrane additives and plasticizers used in the polymeric membranes preparation were selected taking into account the sensor performance, especially the signal stability and repeatability in time of the sensor responses towards basic standard taste substances (salty, sweet, bitter, acid and umami), as previously shown by Dias et al. [30]. These sensor arrays were homemade and their cost only concerns the price of reagents and materials. Furthermore, these devices may be re-used several times, markedly amortising the low initial investment.

Each mixture was prepared by weighting pre-established masses of each one of the above-mentioned 3 products, which were diluted in tetrahydrofurane (from Sigma) in order to obtain a viscous and homogeneous solution. In the multi-sensor system, after removing the protective caps with the aid of a sharp cutting edge, each membrane was prepared by using a drop-by-drop technique (with a 3-5 min interval to ensure complete solvent evaporation) until a transparent crystalline membrane was obtained.

\subsubsection{E-tongue assays}

The assays with the E-tongue device were carried out at room temperature using aqueous solutions of honey ( $10.00 \mathrm{~g}$ of honey diluted in $50.00 \mathrm{~g}$ of deionized water). Before each analysis, the analytical system (two sensor arrays) and the $\mathrm{Ag} / \mathrm{AgCl}$ reference electrode (Crison, 5241) were carefully washed with deionised water and cleaned with an adsorbent paper and then submersed into the stirred aqueous sample solution and allowed to stabilise during $7 \mathrm{~min}$ time-period. Finally the potentiometric signals, varying from $-2.0 \mathrm{~V}$ to $+2.0 \mathrm{~V}$, of 20 different sensor membranes, used in duplicate (S1:1-S1:20 and S2:1-S2:20), were recorded for each sample.

\subsection{Statistical analysis}

This work is focused on the classification of honey samples using linear discriminant analysis (LDA, a supervised multivariate statistical method for classification) according to the colour and floral origin of honey. The mathematical models established are linear combinations of the independent variables (E-tongue sensor signals) that allow the best separation of the different groups of honey (dependent variable). The proposed methodology involved two steps: first, the selection of the most informative independent variables (sensors) by using a Simulated Annealing (SA)

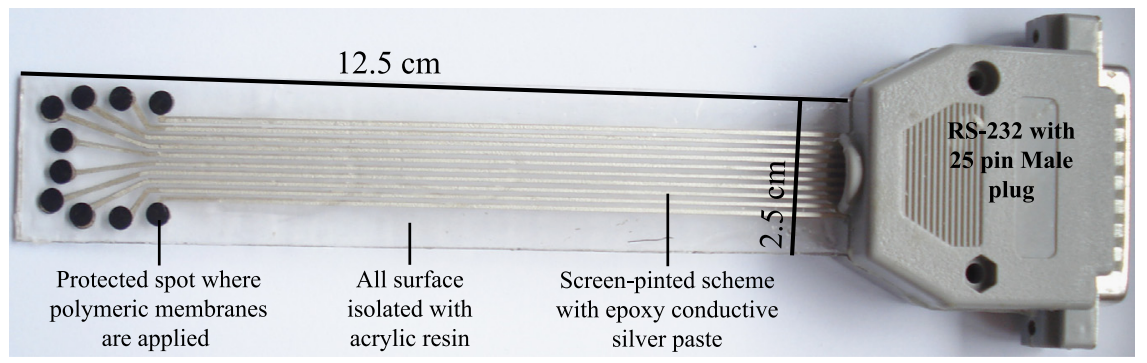

Fig. 1. Multi-sensor system built to use 20 polymeric membranes for potentiometric analysis. 
Table 1

Polymeric membrane compositions of the sensors applied into the E-tongue multi-sensor arrays.

\begin{tabular}{|c|c|c|c|c|c|}
\hline Plasticizer & Additive & ID no. ${ }^{a}$ & Additive (\%) & Plasticizer (\%) & PVC $^{\text {b }}(\%)$ \\
\hline \multirow[t]{4}{*}{ Bis(1-butylpentyl) adipate } & Octadecylamine & $\mathrm{S} 1: 1$ or $\mathrm{S} 2: 1$ & 2.99 & 65.02 & 31.99 \\
\hline & Oleyl alcohol & $\mathrm{S} 1: 2$ or $\mathrm{S} 2: 2$ & 2.96 & 65.00 & 32.04 \\
\hline & Methyltrioctylammonium chloride & $\mathrm{S} 1: 3$ or $\mathrm{S} 2: 3$ & 3.00 & 65.01 & 31.99 \\
\hline & Oleic acid & $\mathrm{S} 1: 4$ or $\mathrm{S} 2: 4$ & 3.00 & 65.04 & 31.96 \\
\hline \multirow[t]{4}{*}{ Dibutyl sebacate } & Octadecylamine & $\mathrm{S} 1: 5$ or $\mathrm{S} 2: 5$ & 3.02 & 65.11 & 31.87 \\
\hline & Oleyl alcohol & S1:6 or S2:6 & 2.96 & 65.02 & 32.02 \\
\hline & Methyltrioctylammonium chloride & $\mathrm{S} 1: 7$ or $\mathrm{S} 2: 7$ & 3.00 & 64.91 & 32.10 \\
\hline & Oleic acid & $\mathrm{S} 1: 8$ or $\mathrm{S} 2: 8$ & 2.99 & 64.93 & 32.09 \\
\hline \multirow[t]{4}{*}{ 2-Nitrophenyl-octylether } & Octadecylamine & $\mathrm{S} 1: 9$ or $\mathrm{S} 2: 9$ & 2.98 & 64.68 & 32.34 \\
\hline & Oleyl alcohol & $\mathrm{S} 1: 10$ or $\mathrm{S} 2: 10$ & 3.00 & 65.10 & 31.90 \\
\hline & Methyltrioctylammonium chloride & $\mathrm{S} 1: 11$ or $\mathrm{S} 2: 11$ & 2.99 & 65.00 & 32.00 \\
\hline & Oleic acid & $\mathrm{S} 1: 12$ or $\mathrm{S} 2: 12$ & 3.01 & 65.04 & 31.95 \\
\hline \multirow[t]{4}{*}{ Tris(2-ethylhexyl)phosphate } & Octadecylamine & $\mathrm{S} 1: 13$ or $\mathrm{S} 2: 13$ & 2.96 & 64.99 & 32.05 \\
\hline & Oleyl alcohol & $\mathrm{S} 1: 14$ or $\mathrm{S} 2: 14$ & 2.97 & 65.04 & 32.00 \\
\hline & Methyltrioctylammonium chloride & $\mathrm{S} 1: 15$ or $\mathrm{S} 2: 15$ & 3.00 & 64.99 & 32.01 \\
\hline & Oleic acid & $\mathrm{S} 1: 16$ or $\mathrm{S} 2: 16$ & 2.98 & 65.04 & 31.98 \\
\hline \multirow[t]{4}{*}{ Dioctyl phenylphosphonate } & Octadecylamine & $\mathrm{S} 1: 17$ or $\mathrm{S} 2: 17$ & 3.12 & 64.85 & 32.03 \\
\hline & Oleyl alcohol & $\mathrm{S} 1: 18$ or $\mathrm{S} 2: 18$ & 2.95 & 65.04 & 32.01 \\
\hline & Methyltrioctylammonium chloride & $\mathrm{S} 1: 19$ or $\mathrm{S} 2: 19$ & 2.99 & 64.69 & 32.32 \\
\hline & Oleic acid & $\mathrm{S} 1: 20$ or $\mathrm{S} 2: 20$ & 3.01 & 65.11 & 31.88 \\
\hline
\end{tabular}

a Identification number.

b Polyvinyl chloride.

Table 2

Floral and colour commercial honey samples classification according to reference melissopalynological and Pfund-colour group analysis.

\begin{tabular}{|c|c|c|c|c|c|c|c|}
\hline \multirow[t]{2}{*}{ Sample } & \multirow[t]{2}{*}{ Harvest year } & \multicolumn{3}{|c|}{ Pollen quantitative spectrum ${ }^{\mathrm{a}}$ (abundance, \%) } & \multirow[t]{2}{*}{ mm Pfund } & \multicolumn{2}{|l|}{ Classification } \\
\hline & & 1st pollen & 2nd pollen & 3rd pollen & & Floral origin & Colour group \\
\hline 1 & 2011 & Ech $53 \%$ & Pru 22\% & Foe $16 \%$ & 32 & Ech & White \\
\hline 2 & 2011 & Ech $71 \%$ & Pru 15\% & Lav 10\% & 16 & Ech & Extra white \\
\hline 3 & 2011 & Ech $73 \%$ & Tri $10 \%$ & Lav $5 \%$ & 27 & Ech & White \\
\hline 4 & 2011 & Ech $79 \%$ & Lav $12 \%$ & Rub 8\% & 26 & Ech & White \\
\hline 5 & 2011 & Ech $60 \%$ & Lav $17 \%$ & Tri $14 \%$ & 32 & Lav & White \\
\hline 6 & 2010 & Ech $34 \%$ & Rub $32 \%$ & Lav $19 \%$ & 26 & Lav & White \\
\hline 7 & 2010 & Ech $38 \%$ & Rub 25\% & Lav $19 \%$ & 33 & Lav & White \\
\hline 8 & 2009 & Rub 39\% & Lav $19 \%$ & Tri $11 \%$ & 20 & Lav & White \\
\hline 9 & 2010 & Ech $39 \%$ & Lav $21 \%$ & Rub 20\% & 21 & Lav & White \\
\hline 10 & 2010 & Ech $39 \%$ & Rub 26\% & Lav $21 \%$ & 33 & Lav & White \\
\hline 11 & 2011 & Ech $36 \%$ & Lav $31 \%$ & Tri $21 \%$ & 33 & Lav & White \\
\hline 12 & 2010 & Lav 31\% & Ech $29 \%$ & Pru 22\% & 28 & Lav & White \\
\hline 13 & 2009 & Lav $32 \%$ & Cas $19 \%$ & Rub 13\% & 32 & Lav & White \\
\hline 14 & 2011 & Lav 39\% & Ech $39 \%$ & Euc 7\% & 27 & Lav & White \\
\hline 15 & 2009 & Lav $45 \%$ & Ech $25 \%$ & Rub 20\% & 27 & Lav & White \\
\hline 16 & 2011 & Lav $45 \%$ & Ech $31 \%$ & Tri $7 \%$ & 16 & Lav & Extra white \\
\hline 17 & 2009 & Lav $47 \%$ & Cas $17 \%$ & Ech $8 \%$ & 28 & Lav & White \\
\hline 18 & 2009 & Lav $49 \%$ & Rub 20\% & Ech $10 \%$ & 27 & Lav & White \\
\hline 19 & 2011 & Lav $56 \%$ & Leo $20 \%$ & Rub 15\% & 22 & Lav & White \\
\hline 20 & 2011 & Lav $67 \%$ & Eri $12 \%$ & Rub $12 \%$ & 31 & Lav & White \\
\hline 21 & 2010 & Ech $53 \%$ & Rub $12 \%$ & Cas $10 \%$ & 39 & Ech & Amber \\
\hline 22 & 2009 & Ech $53 \%$ & Cas $16 \%$ & Lav $8 \%$ & 40 & Ech & Amber \\
\hline 23 & 2010 & Ech $58 \%$ & Cas $7 \%$ & Rub 7\% & 45 & Ech & Amber \\
\hline 24 & 2011 & Ech $70 \%$ & Pru 13\% & Cas $10 \%$ & 47 & Ech & Amber \\
\hline 25 & 2009 & Ech $70 \%$ & Lav $10 \%$ & Pru $8 \%$ & 49 & Ech & Amber \\
\hline 26 & 2010 & Ech $49 \%$ & Rub 16\% & Cas $13 \%$ & 66 & Ech & Amber \\
\hline 27 & 2010 & Ech 53\% & Rub 28\% & Euc 5\% & 67 & Ech & Amber \\
\hline 28 & 2011 & Ech $54 \%$ & Pru 24\% & Lav $10 \%$ & 78 & Ech & Amber \\
\hline 29 & 2011 & Ech $63 \%$ & Pru 26\% & Tri 5\% & 78 & Ech & Amber \\
\hline 30 & 2011 & Ech $54 \%$ & Pru $16 \%$ & Cas $10 \%$ & 100 & Ech & Amber \\
\hline 31 & 2011 & Ech 58\% & Cas $26 \%$ & Tri 13\% & 103 & Ech & Amber \\
\hline 32 & 2011 & Lav 38\% & Ech $30 \%$ & Rub 18\% & 35 & Lav & Amber \\
\hline 33 & 2009 & Lav 33\% & Rub 20\% & Thy $18 \%$ & 36 & Lav & Amber \\
\hline 34 & 2011 & Lav $46 \%$ & Pru 40\% & Tri $8 \%$ & 42 & Lav & Amber \\
\hline 35 & 2009 & Lav 31\% & Pru 29\% & Ech $20 \%$ & 51 & Lav & Amber \\
\hline 36 & 2011 & Pru 32\% & Lav $27 \%$ & Ech $26 \%$ & 104 & Lav & Amber \\
\hline 37 & 2011 & Pru 64\% & Ech 15\% & Aca $13 \%$ & 61 & Pru & Amber \\
\hline 38 & 2011 & Pru 50\% & Cas $38 \%$ & Rub 3\% & 63 & Pru & Amber \\
\hline 39 & 2011 & Pru 80\% & Cas 6\% & Euc 6\% & 69 & Pru & Amber \\
\hline 40 & 2009 & Rub 58\% & Aca $22 \%$ & Pru $7 \%$ & 40 & Rub & Amber \\
\hline 41 & 2011 & Rub 47\% & Euc $21 \%$ & Pru 19\% & 59 & Rub & Amber \\
\hline
\end{tabular}


Table 2 (continued)

\begin{tabular}{|c|c|c|c|c|c|c|c|}
\hline \multirow[t]{2}{*}{ Sample } & \multirow[t]{2}{*}{ Harvest year } & \multicolumn{3}{|c|}{ Pollen quantitative spectrum ${ }^{\mathrm{a}}$ (abundance, \%) } & \multirow[t]{2}{*}{ mm Pfund } & \multicolumn{2}{|l|}{ Classification } \\
\hline & & 1st pollen & 2nd pollen & 3rd pollen & & Floral origin & Colour group \\
\hline 42 & 2010 & Rub 62\% & Ech $14 \%$ & Aca $13 \%$ & 60 & Rub & Amber \\
\hline 43 & 2010 & Rub $49 \%$ & Cas $17 \%$ & Gen $11 \%$ & 66 & Rub & Amber \\
\hline 44 & 2009 & Rub $52 \%$ & Eri $12 \%$ & Pru 11\% & 74 & Rub & Amber \\
\hline 45 & 2009 & Rub 50\% & Cas $27 \%$ & Tri $8 \%$ & 76 & Rub & Amber \\
\hline 46 & 2009 & Rub 69\% & Pru 7\% & Eri $3 \%$ & 81 & Rub & Amber \\
\hline 47 & 2009 & Rub 49\% & Tri $17 \%$ & Cas $14 \%$ & 101 & Rub & Amber \\
\hline 48 & 2010 & Rub 57\% & Tri $22 \%$ & Eri 10\% & 111 & Rub & Amber \\
\hline 49 & 2010 & Rub 50\% & Lav $14 \%$ & Cas $12 \%$ & 113 & Rub & Amber \\
\hline 50 & 2009 & Rub 51\% & Cas $11 \%$ & Lav 6\% & 114 & Rub & Amber \\
\hline 51 & 2009 & Cas $92 \%$ & Eri 1\% & Pru $1 \%$ & 119 & Cas & Dark \\
\hline 52 & 2011 & Cas $95 \%$ & Rub 5\% & - & 134 & Cas & Dark \\
\hline 53 & 2011 & Cas $91 \%$ & Rub 5\% & Tri 4\% & 147 & Cas & Dark \\
\hline 54 & 2011 & Cas $90 \%$ & Euc 6\% & Ech 3\% & 176 & Cas & Dark \\
\hline 55 & 2009 & Eri $50 \%$ & Cas $28 \%$ & Pru 8\% & 127 & Eri & Dark \\
\hline 56 & 2009 & Eri 53\% & Cas $15 \%$ & Rub 13\% & 129 & Eri & Dark \\
\hline 57 & 2009 & Eri 61\% & Tri $17 \%$ & Pru $12 \%$ & 141 & Eri & Dark \\
\hline 58 & 2011 & Eri 56\% & Tri 23\% & Pru 8\% & 171 & Eri & Dark \\
\hline 59 & 2010 & Eri $54 \%$ & Cas $19 \%$ & Rub 8\% & 172 & Eri & Dark \\
\hline 60 & 2009 & Eri $82 \%$ & Cas $12 \%$ & Ech 6\% & 193 & Eri & Dark \\
\hline 61 & 2011 & Eri $54 \%$ & Cas $26 \%$ & Ech $7 \%$ & 199 & Eri & Dark \\
\hline 62 & 2011 & Eri $63 \%$ & Rub 19\% & Cas $15 \%$ & 201 & Eri & Dark \\
\hline 63 & 2010 & Rub 50\% & Ech $17 \%$ & Cas $16 \%$ & 142 & Rub & Dark \\
\hline 64 & 2011 & Rub 61\% & Ech $14 \%$ & Eri 13\% & 147 & Rub & Dark \\
\hline 65 & 2009 & Rub 49\% & Cas $22 \%$ & Lav 10\% & 196 & Rub & Dark \\
\hline
\end{tabular}

${ }^{\text {a }}$ Aca - Acacia sp.; Cas - Castanea sp.; Ech - Echium sp.; Eri - Erica sp.; Euc - Eucalyptus sp.; Foe - Foeniculum sp.; Gen - Genista sp.; Lav - Lavandula sp.; Pru - Prunus sp.; Rub - Rubus sp.; Thy - Thymus sp.; and Tri - Trifolium sp.

meta-heuristic variable selection algorithm, which is a key task considering that signal profiles recorded by E-tongue devices usually show a high multicollinearity degree; then, the performance evaluation of the selected models regarding sample classification was carried out using a leave-one-out (LOO) crossvalidation technique to avoid overoptimistic correct classification results. This methodology enabled to evaluate the model's prediction performance by removing a set of $n$ samples from the database and then predict their rank with the LDA model obtained with the remaining $n-1$ samples. This process was repeated $n$ times, to obtain the classification errors of all the samples, and the overall sum of errors for each test divided by $n$.

The subsets of independent variables selected by applying the SA algorithm allowed obtaining LDA models with fewer sensors, eliminating redundant sensor that had a similar contribution to the differences between groups, increasing the accuracy of the prediction. The final model selected will be simpler and easier to interpret, allowing the best prediction performance with the minimum number of sensors. The algorithm was programmed to give the best model for each subset, using 2-20 sensors selected among the 40 recorded potentiometric signals (which resulted in 19 models) and after 10,000 attempts. The subset range was set between 2 and 20 with the purpose of achieving the simplest model (with the minimum number of sensors) and also considering the number of honey samples belonging to each colour group. The number of attempts was set equal to 10,000 as this value enabled to reach always the same best solution (type of sensors included in the model) for subsets with higher number of variables. The quality criterion ccr12 (Roy's first root statistical coefficient) was used to assess the goodness of fitting between the dependent variable (colour or types of monofloral honey) and each one of the 19 subsets of sensors chosen. Maximising this criterion is equivalent to the maximisation of the first root Roy, which is the ratio between the unexplained and the explained variance for the first discriminant function (conceptually equivalent to the value $\mathrm{F}$ ratio in the analysis of variance) [31,32]. All statistical analysis was performed using Subselect [31,32] and
MASS [33] packages of the open source statistical program $R$ (version 2.15.1).

\section{Results and discussion}

\subsection{Honey colour and floral origin classifications}

When the 65 honey samples were analysed by UV-vis spectrophotometry and the absorbances of aqueous diluted samples transformed into mm Pfund according to Eq. (1), values from 15.9 up to $204.2 \mathrm{~mm}$ Pfund were determined, enabling honey to be classified according to the honey colour scale $[27,28]$. The results showed that samples had colours between extra white and dark amber: 2 extra white, 18 white, 9 extra light amber, 14 light amber, 7 amber and 15 dark amber honey samples.

Using the colour classification methodology proposed in this work (Section 2.1), samples were split into 3 main groups as follows: 20 white samples with $\mathrm{mm}$ Pfund lower than 34 (between 15.9 and $33.3 \mathrm{~mm}$ Pfund); 30 amber samples with $\mathrm{mm}$ Pfund ranging from 34.8 to $113.6 \mathrm{~mm}$ Pfund; and 15 dark samples with $\mathrm{mm}$ Pfund greater than $118.8 \mathrm{~mm}$ Pfund. This procedure is needed as a first data treatment step for honey analysis with the potentiometric E-tongue due to the wide colour variability observed in honey samples, which is mainly influenced by the honey floral origin.

Concerning the floral origin of each honey sample, it was evaluated using pollen grain identification and count. Globally, for the 65 honey samples analysed, it was possible to identify 23 different kinds of pollen. By descending order considering their presence in the overall samples, these were: Rubus sp., Lavandula sp., Prunus sp., Echium sp., Castanea sp., Trifolium sp. and Erica sp. (present in more than $44 \%$ of the samples), followed by Eucalyptus sp., Leontodeon sp., Thymus sp., Cytisus sp., Acacia sp. and Pinus sp. (detected in 12-34\% of the samples) and finally the other type of pollens, Sandix sp., Foeniculum sp., Helianthus sp., Genista sp., Tilia sp., Mentha sp., Persea sp., Medicago sp., Crepis sp. and Mimosaceae 
sp. (identified in less than $10 \%$ of the samples). These different identified pollens were already reported for other Portuguese honey [34]. The pollinic profiles determined showed that in 61 honey samples it was possible to identify between 4 and 9 different pollens, in 3 honey samples 3 different pollens were detected and, finally, in only 1 sample 2 different pollens were found. Independently of their colours and based on their pollinic profiles and on the relative abundance of each pollen [34,35], the 65 honey samples were classified as Castanea, Echium, Erica, Lavandula, Prunus and Rubus monofloral honey and, further split according to the 3 main colour groups previously defined: white, amber and dark. Table 2 presents information concerning harvest year, colour classification and pollinic profile (considering only the 3 pollens most predominante) of the honey samples analysed, which enables a detailed overview of the wide variability found for these two important honey characteristics.

These results, as expected, also confirm the high floral origin variability of the honey samples analysed in this work. Only the Castanea honey showed lower variability in the pollinic profile, since the main pollen (Castanea sp.) represented $90-95 \%$ of prevalence. For the other monofloral honey, the main pollen varied over a wider range of percentages: Echium between $49 \%$ and $79 \%$; Erica between $50 \%$ and $82 \%$; Lavandula between $17 \%$ and $67 \%$; Prunus between 49\% and 80\%; and Rubus between 47\% and 69\%.

\subsection{E-tongue results}

\subsubsection{E-tongue signals profiles of honey samples}

In the total, 65 assays were carried out, each providing 40 potentiometric signals (20 different sensor membranes used in duplicate: S1:1-S1:20 and S2:1-S2:20). Fig. 2 shows the potentiometric signal's box-plots for each sensor and monofloral honey samples grouped according to colour (white, amber and dark). The potentiometric signals varied from $+0.09 \mathrm{~V}$ to $+0.23 \mathrm{~V}$ for all sensors included in the E-tongue, avoiding the need of data scaling. Fig. 2 shows that slight differences in signal intensities occur for some sets of sensors, implying the need of a variable

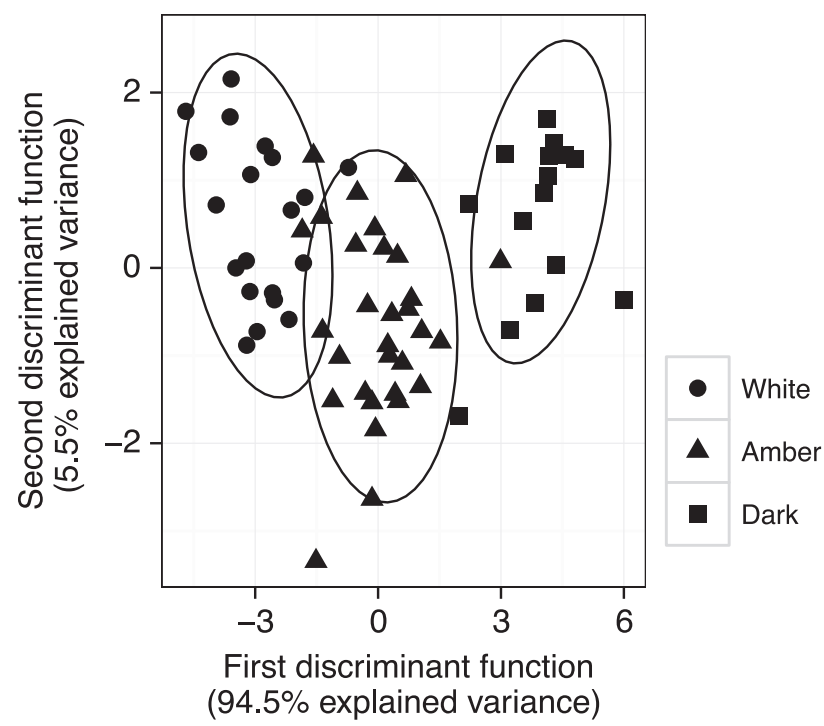

Fig. 3. Linear discriminant analysis between the three colour groups associated with the primary selection of samples. The ellipses in each group are associated with the confidence interval of $90 \%$, assuming a multivariate normal distribution.

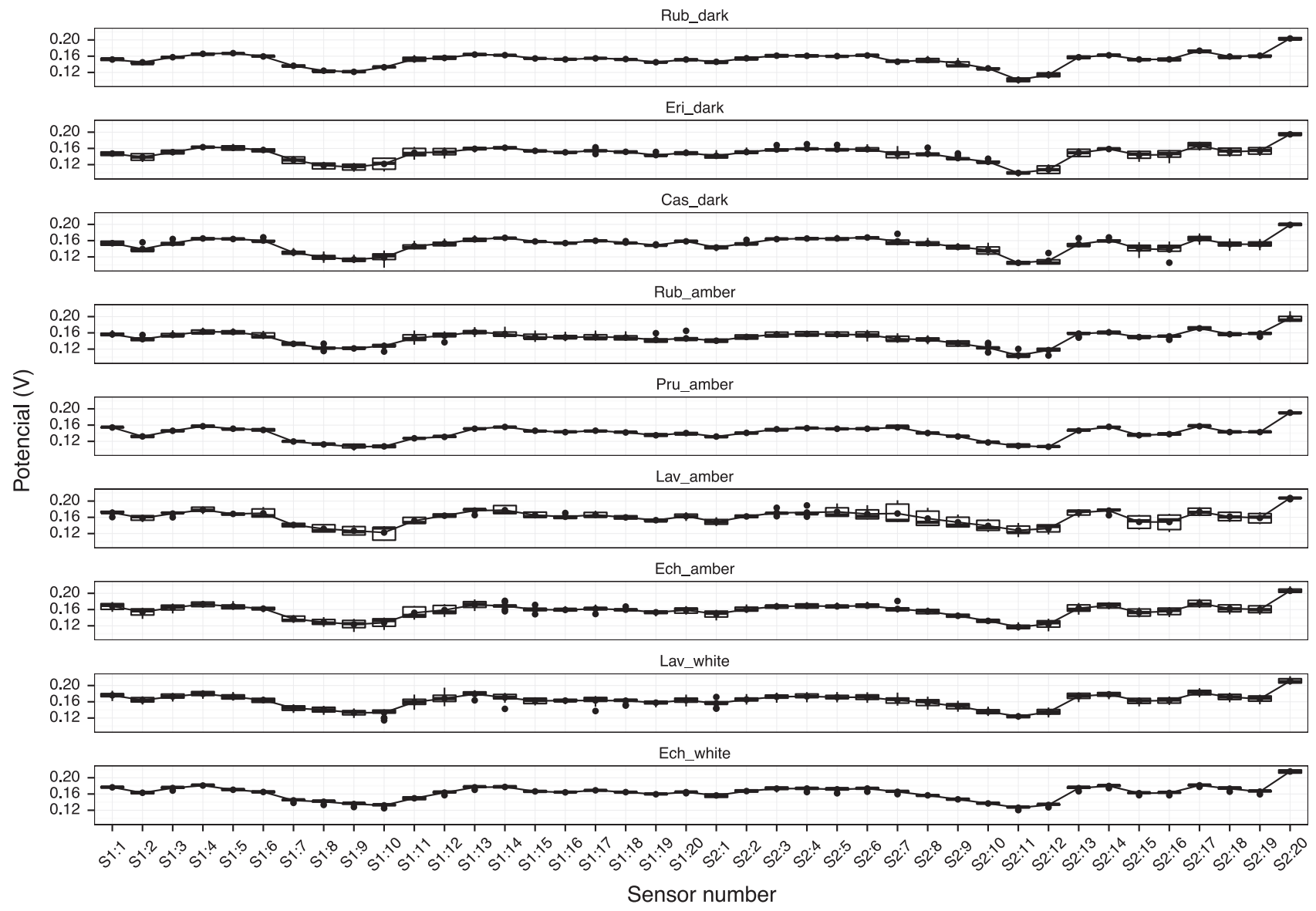

Fig. 2. Potentiometric signal's box-plots for each sensor and monofloral honey grouped according to colour (white, amber and dark). 
selection procedure to choose the most relevant signals for establishing the best predictive LDA model.

\subsubsection{E-tongue analysis}

The classification performance of the E-tongue was evaluated considering colour and floral origin factors. A two-step procedure was adopted, using the potentiometric signal data and LDA, with the sensors subsets selected by the SA algorithm. First honey samples were classified according to colour, independently of their floral origin; and after, for each of the previous groups, samples were discriminated considering their floral origin. This procedure was adopted because it was not possible (data not shown) to establish a predictive LDA model that would allow a leave-one-out cross-validation satisfactory classification ( $\leq 45 \%$ of correct classifications) when using simultaneously the 65 honey samples of the 6 monofloral honeys.

The results showed that, the potentiometric E-tongue developed allowed a satisfactory discrimination of honey samples into the 3 main colour groups established (white, amber and dark honey) using LDA based on a subset of 13 sensors selected by the SA algorithm (S1:3; S1:5; S1:7; S1:14; S1:16; S1:20; S2:2; S2:11; S2:14; S2:16; S2:17; S2:18 and S2:19) (Fig. 3), which may confirm that there is similarity among the potentiometric signal profiles of the samples of each group. Although colour is a visual characteristic, the capability of the E-tongue to distinguish honey samples according to their colours may be due to the different responses of the sensors device towards the different physicochemical and matrix composition of the different monofloral honey samples studied [36-40].

For that, two discriminant functions were established explaining $100 \%$ of the total original data variance (94.5\% and $5.5 \%$ ). The first function enables the discrimination of the three colour groups in an expected lighter-to-darkness tone sequence, being white honey placed in the negative region, amber honey in the middle zone (corresponding to scores near to zero) and dark honey in the positive region. The sensors selected covered all the plasticizers and additives used in the membrane composition. Both original data and LOO cross-validation (Table 3) classifications allowed achieving 91\% of the honey samples correctly classified (only 6 samples of the 65 honey samples were misclassified), being white and dark honey classifications the most accurate. These results are quite satisfactory, especially if it is taken into account that 6 different floral origins were identified in the honey samples studied and also that they were collected during a 3-year time period.

The E-tongue gave very satisfactory results regarding the discrimination of monofloral honey according to their floral origin, within each honey colour group previously defined (Figs. 4-6), without showing any correlation with their harvest years.

Indeed, for white, amber and dark colour groups the E-tongue enabled $100 \%$ correct classifications with a LDA leave-one-out cross-validation procedure. For the white honey group, containing Lavandula and Echium monofloral honey (16.3-33.3 mm Pfund and 15.9 up to $32 \mathrm{~mm}$ Pfund, respectively), one linear discriminant function (explaining $100 \%$ of the data variance) was established

Table 3

LDA contingency matrix for the monofloral honey samples colour classification based on the E-tongue signals recorded (results from leave-one-out cross-validation procedure)

\begin{tabular}{llccl}
\hline \multirow{2}{*}{$\begin{array}{l}\text { Actual honey } \\
\text { colour group }\end{array}$} & \multicolumn{2}{l}{ Predicted honey colour group } & \multirow{2}{*}{ Sensitivity (\%) } \\
\cline { 2 - 4 } & White & Amber & Dark & \\
\hline White & 19 & 1 & 0 & 95 \\
Amber & 3 & 26 & 1 & 87 \\
Dark & 0 & 1 & 14 & 93 \\
\hline
\end{tabular}

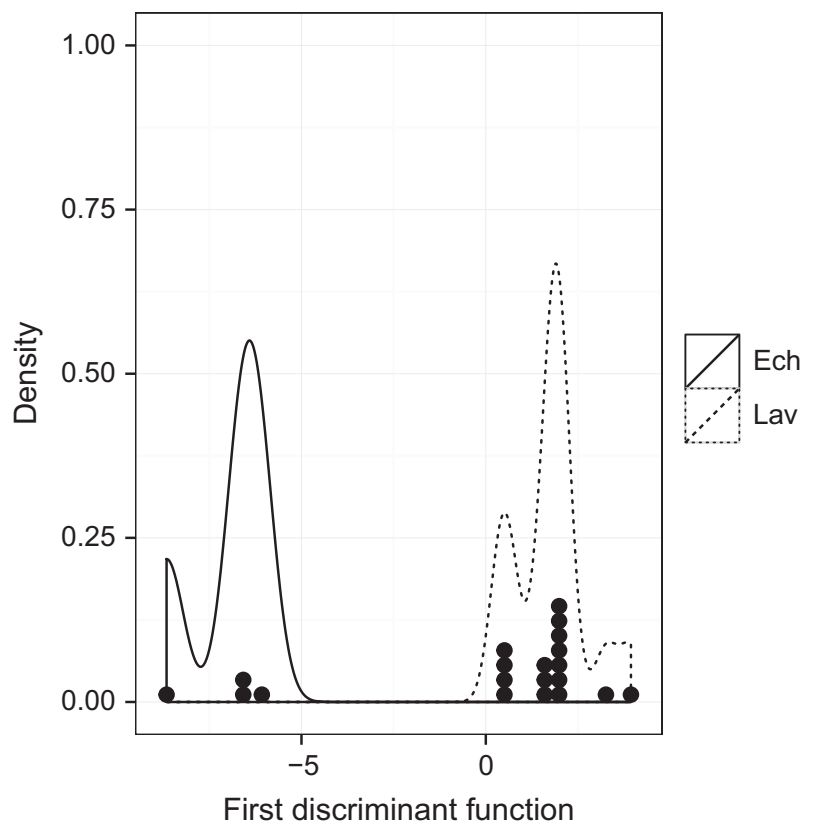

Fig. 4. Frequency distribution of the data referring to the first discriminant function classification of two groups of white-coloured monofloral honey: Echium sp. (Ech) and Lavandula sp. (Lav).

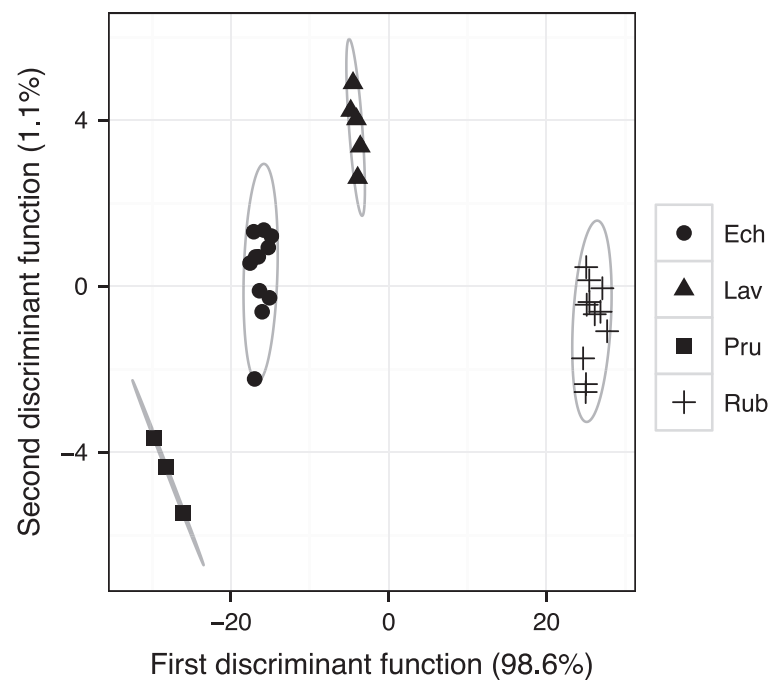

Fig. 5. Linear discriminant analysis of four groups of amber-coloured monoflora honey: Echium sp. (Ech), Lavandula sp. (Lav), Prunus sp. (Pru) and Rubus sp. (Rub). The ellipses in each group are associated with the confidence interval of $95 \%$, assuming a multivariate normal distribution.

based on the potentiometric signal data recorded by a set of 6 sensors (S1:1, S1:3, S1:10, S2:2, S2:3, S2:16), which were selected using the SA algorithm. Regarding the amber honey group, which included Echium, Lavandula, Prunus and Rubus monofloral honey (38.9-103.2 mm Pfund; 34.8-103.5 mm Pfund; $60.8-69.0 \mathrm{~mm}$ Pfund and 40.0 up to $113.6 \mathrm{~mm}$ Pfund, respectively), three linear discrminant functions, with 16 sensors (S1:6, $\mathrm{S} 1: 7, \mathrm{~S} 1: 8, \mathrm{~S} 1: 10, \mathrm{~S} 1: 12, \mathrm{~S} 1: 14, \mathrm{~S} 1: 17, \mathrm{~S} 2: 1, \mathrm{~S} 2: 4, \mathrm{~S} 2: 8, \mathrm{~S} 2: 12, \mathrm{~S} 2: 14$, $\mathrm{S} 2: 15, \mathrm{~S} 2: 16, \mathrm{~S} 2: 18, \mathrm{~S} 2: 19$ ), were needed (explaining $98.6 \%, 1,1 \%$ and $0.3 \%$ of the original data total data variance, respectively). Finally, for the dark honey group, containing Castanea, Erica and Rubus monofloral honey (118.8-175.6 mm Pfund; 126.6$201.2 \mathrm{~mm}$ Pfund; and 142.2 up to $196.0 \mathrm{~mm}$ Pfund, respectively), two linear discriminant functions, based on the signals of 7 sensors (S1:12, S1:15, S1:18, S2:7, S2:9, S2:13, S2:19), were established 


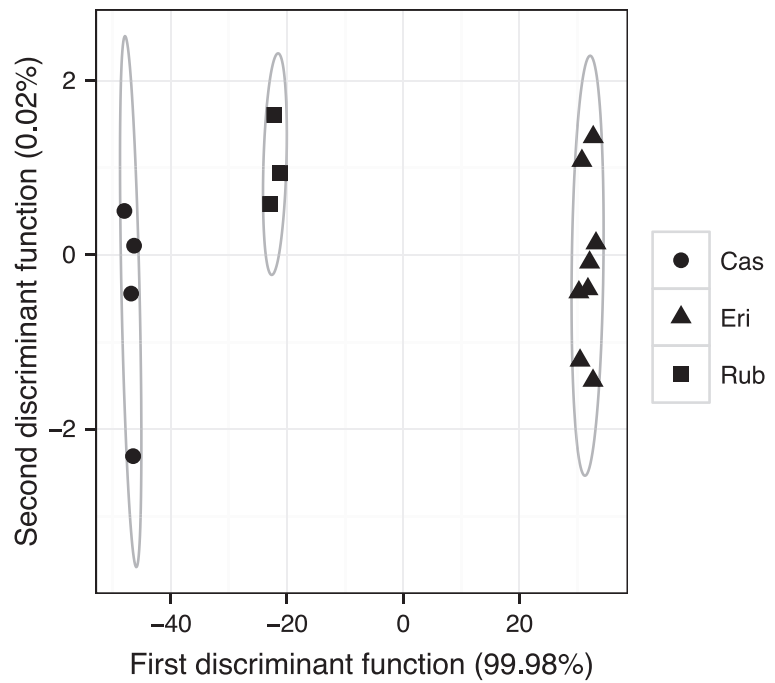

Fig. 6. Linear discriminant analysis of three groups of dark-coloured monofloral honey: Castanea sp. (Cas), Erica sp. (Eri) and Rubus sp. (Rub). The ellipses in each group are associated with the confidence interval of $95 \%$, assuming a multivariate normal distribution.

(accounting for $99.98 \%$ and $0.02 \%$ of the original data total variance, respectively).

The analysis of the type of sensors selected for each colour group, considering the plasticizer plus additive combination, shows that, in general, all plasticizers and additives were used with the same frequency, which means that all the combinations used were adequate for extracting the most relevant information from the honey samples. Furthermore, for white and amber groups, the variable selection algorithm enabled the inclusion of repeated sensors, with the same membrane composition, in the discrimination models, each from one of the two sensor arrays used, which is in accordance with the findings previous reported [41], showing that the inclusion of repeated sensors in multivariate analysis can improve model performance. Indeed, from a modelling point of view, duplicate potentiometric sensors can be seen as independent variables considering that slight variations of the membrane composition and physical properties (transparency and porosity) may occur when a drop-by-drop technique is used for membrane preparation, resulting in slightly different signal profiles recorded by two sensor replicas. Also, some the sensors selected for two colour groups, by applying the SA algorithm, were equal, namely for white and amber groups (membrane nos. 1, 10 and 16) and amber and dark groups (membrane nos. 7, 12, 15, 18 and 19). The selection of these same sensors may be due to the presence of honey samples with the same floral origin belonging to different honey colour groups. The use of the meta-heuristic SA algorithm for variable selection enabled the identification and selection of a minimum set of sensors required to fully discriminate monofloral honey samples according to their floral origin, after colour honey classification. Moreover, compared with previous reported applications of E-tongue for floral origin classification of honey, the performance of the proposed potentiometric E-tongue is similar $[10,22]$ or quite superior $[6,20,21,26]$.

From a global point of view, it is interesting to note that the inclusion of more sensors (variables) in the linear discriminant functions previously described would result in a lower classification performance of the E-tongue, when a LOO cross-validation procedure was applied (data not shown). This fact corroborates the importance of using an adequate variable selection algorithm that could deal simultaneously with co-linearity issues between potentiometric signal profiles and the selection of the data that provide a truly chemical fingerprint of the samples. It is known that the choice of sensors is a key principle in designing E-tongues [42]. This potential may be enhanced if a robust variable selection algorithm, such as the SA meta-heuristic algorithm, is used together with traditional LDA.

Finally, it should be noted that this novel approach (sample split according to honey colour coupled with cross-sensitivity potentiometric E-tongue) showed a similar [10,22] or better $[6,20,21,26]$ performance concerning floral origin honey sample classification when compared with previous works. Moreover, the proposed methodology is successfully applied to honey samples that, although being classified as monofloral, had a confirmed broader pollinic composition and have been collected during a 3 -year period, which compared with the previous works represents a sampling procedure with greater intrinsic variability.

On the whole, these results show the usefulness of the device proposed in this work for potential use at analytical laboratory level for floral origin honey classification.

\section{Conclusion}

The combined strategy adopted in this work, coupling a prior split step of honey sample by colour determined by spectrophotometry analysis together with a potentiometric E-tongue enabled monofloral honey discrimination according to floral origin (100\% of correct classifications for LOO cross-validation), for samples with high colour and pollen profile variabilities. The quality of the results achieved with the E-tongue designed and built in this work, with cross-sensitivity lipid membranes, shows that the selection and incorporation of cross-sensitivity lipidic membranes into the sensor-array provided a useful and informative chemical fingerprint from the monofloral honey samples, enabling their floral origin discrimination.

\section{References}

[1] J.W. White Jr., L.W. Doner, Honey Composition and Properties, Beekeeping in the United States, Agric Handbook, 198082-91.

[2] M.J. Diez, C. Andres, A. Terrab, Int. J. Food Sci. Technol. 39 (2004) 167-176.

[3] M.L. Al, D. Daniel, A. Moise, O. Bobis, L. Laslo, S. Bogdanov, Food Chem. 112 (2009) 863-867.

[4] J.W. White, Assoc. Off. Anal. Chem. J. 67 (1984) 1129-1131.

[5] M. Viuda-Martos, Y. Ruiz-Navajas, J.M. Zaldivar-Cruz, V. Kuri, J. FernándezLópez, Á.A. Carbonell-Barrachina, J.Á. Pérez-Álvarez, Int. J. Food Sci. Technol. 45 (2010) 1111-1118.

[6] I. Escriche, M. Kadar, E. Domenech, L. Gil-Sánchez, J. Food Eng. 109 (2012) 449-456.

[7] I. Escriche, M. Kadar, M. Juan-Borrás, E. Domenech, Food Chem. 142 (2014) 135-143.

8] G. Scandurra, G. Tripodi, A. Verzera, J. Food Eng. 119 (2013) 738-743.

[9] E. Corbella, D. Cozzolino, Chil. J. Agric. Res. 68 (2008) 102-107.

[10] N. Major, K. Marković, M. Krpan, G. Šarić, M. Hruškar, N. Vahčić, Talanta 85 (2011) 569-574.

[11] C. Acquarone, P. Buera, B. Elizalde, Food Chem. 101 (2007) 695-703.

[12] G. Beretta, P. Granata, M. Ferrero, M. Orioli, R.M. Facino, Anal. Chim. Acta 533 (2005) 185-191.

[13] N. Gheldof, X.H. Wang, N.J. Engeseth, J. Agric. Food Chem. 50 (2002) $5870-5877$.

[14] Z. Wei, J. Wang, W. Liao, J. Food Eng. 96 (2010) 469-479.

[15] L. Castro-Vázquez, M.C. Díaz-Maroto, M.A. González-Viñas, M.S. Pérez-Coello, Food Chem. 112 (2009) 1022-1030.

[16] L.F. Cuevas-Glory, J.A. Pino, L.S. Santiago, E. Sauri-Duch, Food Chem. 103 (2007) $1032-1043$.

[17] I. Escriche, M. Kadar, M. Juan-Borrás, E. Domenech, Food Res. Int. 44 (2011) 1504-1513.

[18] J.M. Camiña, R.G. Pellerano, E.J. Marchevsky, Curr. Anal. Chem. 8 (2012) $408-425$.

[19] C.H. Latorre, R.M.P. Crecente, S.G. Martín, J.B. García, Food Chem. 141 (2013) $3559-3565$

[20] L.A. Dias, A.M. Peres, M. Vilas-Boas, M.A. Rocha, L. Estevinho, A.A.S.C. Machado, Microchim. Acta 163 (2008) 97-102.

[21] Z. Wei, J. Wang, W. Liao, J. Food Eng. 94 (2009) 260-266.

[22] A. Zakaria, A.Y. Md Shakaff, M.J. Masnan, M.N. Ahmad, A.H. Adom, M.N. Jaafar, S.A. Ghani, A.H. Abdullah, A.H.A. Aziz, L.M. Kamarudin, N. Subari, N.A. Fikri, Sensors 11 (2011) 7799-7822. 
[23] K. Tiwari, B. Tudu, R. Bandyopadhyay, A. Chatterjee, J. Food Eng. 117 (2013) 205-210.

[24] Z. Wei, J. Wang, Electrochim. Acta 56 (2011) 4907-4915.

[25] P.A. Ulloa, R. Guerra, A.M. Cavaco, A.M.R. Costa, A.C. Figueira, A.F. Brigas, Comput. Electron. Agric. 94 (2013) 1-11.

[26] E. Garcia-Breijo, J. Garrigues, L.G. Sanchez, N. Laguarda-Miro, Sensors 13 (2013) 10418-10429.

[27] I.C.F.R. Ferreira, E. Aires, J.C.M. Barreira, L.M. Estevinho, Food Chem. 114 (2009) $1438-1443$.

[28] USDA Agricultural Marketing Service, United States Standards for Grades of Extracted Honey, USDA, Washington DC, May 23, 1985.

[29] J. Louveaux, A. Maurizio, G. Vorwohl, Bee World 59 (1978) 139-157.

[30] L.A. Dias, A.M. Peres, A.C.A. Veloso, F.S. Reis, M. Vilas-Boas, A.A.S.C. Machado, Sens. Actuat. B 136 (2009) 209-217.

[31] J. Cadima, J.O. Cerdeira, M. Minhoto, Comput. Stat. Data Anal. 47 (2004) 225-236.

[32] J. Cadima, J.O. Cerdeira, P.D. Silva, M. Minhoto, The subselect R package, 2012. 〈http://cran.r-project.org/web/packages/subselect/vignettes/subselect.pdf〉.
[33] W.N. Venables, B.D. Ripley, Modern Applied Statistics with S, fourth ed. Springer, New York, ISBN 0-387-95457-0, 2002.

[34] S.M.A. Pires, T. Rodrigues, A. Rocha, A. Pajuelo, O. Pereira, R. Port. Zoot. 1 (2005) 87-99.

[35] W. Von Der Ohe, L.P. Oddo, M.L. Piana, M. Morlot, P. Martin, Apidologie 35 (2004) S18-S25.

[36] V. Baltrusaityte, P. Petras, V. Ceksteryte, Food Chem. 101 (2007) 502-514.

37] E.F. Boffo, L.A. Tavares, A.C.T. Tobias, M.M.C. Ferreira, A.G. Ferreira, LWT - Food Sci. Technol. 49 (2012) 55-63.

[38] I. Jasicka-Misiak, A. Poliwoda, M. DereÅ, P. Kafarski, Food Chem. 131 (2012) 1149-1156.

[39] J.J.J. Lacerda, J.S. Santos, S.A. Santos, G.B. Rodrigues, M.L.P. Santos, Quím. Nova 33 (2010) 1022-1026.

[40] Md. I. Khalil, M. Moniruzzaman, L. Boukraâ, M. Benhanifia, Md. A. Islam, Md. N. Islam, S.A. Sulaiman, S.H. Gan, Molecules 17 (2012) 11199-11215.

41] D.P.A. Correia, J.M.C.S. Magalhães, A.A.S.C. Machado, Talanta 67 (2005) 773-782.

[42] J.M. Gutiérrez, Z. Haddi, A. Amari, B. Bouchikhi, A. Mimendia, X. Cetó, M. del Valle, Sens. Actuat. B. 177 (2013) 989-996. 